\section{Brave fish forage more}

Fishing can affect not just the numbers of predators and the behaviour of their prey, but also the distribution of plants at the bottom of the food chain.

Elizabeth Madin, currently at the University of TechnologySydney in Australia, and her colleagues developed a model assuming that a reduction in predators from fishing makes prey species bolder. They are then inclined to forage farther afield.

The authors used the model to accurately predict the spatial distribution of seaweed, which is eaten by the prey fish, on the coral reefs of the northern Line Islands in the Pacific Ocean. Those of Palmyra Atoll, which has seen almost no fishing, had more and larger seaweed patches - indicating less adventurous foraging - than did the intensely fished reefs of Kiritimati Atoll.

Am. Nat. doi:10.1086/657039 (2010)

\section{APPLIED PHYSICS}

\section{Long-lasting memory storage}

A data-storage device based on silver ions embedded in glass could theoretically store 180 gigabytes per square centimetre - about 80 times the capacity of a Blu-ray disc.

Lionel Canioni at the University of Bordeaux in France and his team embedded a three-dimensional matrix of silver ions in light-sensitive glass. The ions fluoresce when hit with a laser. The researchers could control the silver's fluorescence at 16 discrete levels, coding information as varying levels of brightness rather than as the binary 'ones' and 'zeroes' that conventional magnetic storage devices use.

The authors managed to store and retrieve micrometresized pictures of three French Nobel prizewinners. Because glass resists wear from temperature and humidity, the authors say that it could be used for high-density information storage that lasts centuries.

Adv. Mater. doi:10.1002/

adma.201002413 (2010)

\section{IMMUNOLOGY}

\section{How NETs form to capture invaders}

As a first line of defence against microbes, immune cells called neutrophils release webs of uncoiled DNA strands and antimicrobial molecules to ensnare and kill the invaders (pictured). Researchers have identified two enzymes that work together to help to form these traps, known as NETs.

Neutrophils generate reactive oxygen-containing molecules and then undergo cell death, releasing the NETs. Arturo Zychlinsky and his team at the Max Planck Institute for Infection Biology in Berlin tested neutrophil extracts for their ability to unwind DNA and disrupt the cell nucleus key steps in NET formation.

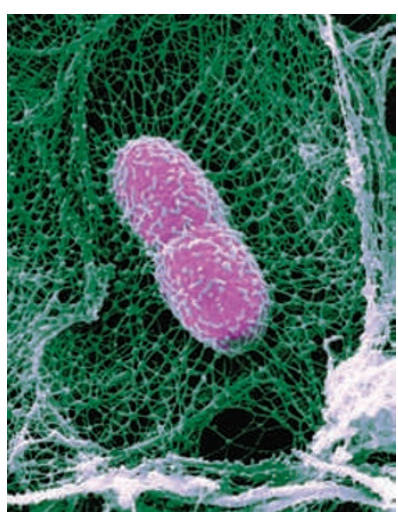

They homed in on two enzymes, neutrophil elastase and myeloperoxidase, that are normally stored in granules. When activated by the oxygencontaining molecules, the enzymes move to the nucleus, where they unwind DNA. J. Cell Biol. doi:10.1083/ jcb.201006052 (2010)

\section{ECOLOGY \\ Climate controls tree growth}

Climatic factors such as rainfall, seasonality and temperature have a greater

COMMUNITY CHOICE

The most viewed papers in science

\title{
The enigma of genes and environment
}

\section{AIGHLY READ \\ on plosgenetics.org 11-24 October}

The interplay between an animal's genes and environment at the molecular level is largely mysterious. Researchers report that, even in a simple yeast model, the

two interact in ways that are difficult to predict.

Barak Cohen and his colleagues at Washington University in St Louis, Missouri, created 32 yeast strains carrying different combinations of four single-nucleotide gene variants that affect how efficiently the yeast forms spores. The strains were generated with two different genetic backgrounds and cultured in eight different growth media. The researchers then tracked changes in spore formation under each condition.

The authors were able to predict the effects of a genetic variant on spore formation, but only after they had accounted for a strain's growth environment and genetic background. This suggests that those studying human genetics and disease need to measure the effects of such factors.

PLoS Genet. 6, e1001144 (2010)

effect on tropical tree growth than logging or soil nutrient levels, a study of Bolivian lowland forests reveals.

Marisol Toledo of the Bolivian Forest Research Institute in Santa Cruz and her colleagues monitored tree growth in 165 one-hectare plots for periods of up to 11 years, and collected data on climate, soil nutrition and disturbance. They found that logging does increase growth, but to a lesser extent than climate, and the effect disappears six years after trees are felled. Nutrient levels in soil do not have a large effect.

Forest growth is projected to slow with climate change, as rainfall is predicted to decrease and seasonality to increase in the tropics. However, these changes could be partially offset by rising temperatures. J. Ecol. doi:10.1111/j.13652745.2010.01741.x (2010)

\section{STRUCTURAL BIOLOGY}

\section{A walk through the genome}

Genes are regulated in numerous ways; one is the addition of methyl groups to histone proteins, which bind and package up DNA. A study of a viral enzyme that methylates histones suggests that it functions by 'walking' from one methylation site to the next.

The enzyme, called vSET, is found in Paramecium bursaria chlorella virus 1 and suppresses gene expression in the host to aid infection. Hua Wei and Ming-Ming Zhou at Mount Sinai School of Medicine in New York determined the three-dimensional structure of the enzyme and studied how it binds to histones. They surmised that vSET has two binding areas, of which only one can bind to the substrate at a time. Once methylation has occurred at the first binding site, vSET is released at the same time that its second binding area attaches to another site. This 'walking' mechanism allows the enzyme to work efficiently across the whole genome, the authors say. Proc. Natl Acad. Sci. USA doi:10.1073/pnas.1009911107 (2010)

\section{$\rightarrow$ NATURE.COM}

For the latest research published by Naturevisit:

www.nature.com/latestresearch 